\title{
Initial Serum Creatine Phosphokinase Level as an Indicator of Severity and Prognosis of Acute Organophosphorus Poisoning - A Retrospective Clinical Study
}

\author{
Ajitha Kesi Chellappan¹, Regi David Sam², Padmakumar Rajashekharan Pillai ${ }^{3}$, Ajith Syamala Narayanan ${ }^{4}$ \\ 1, 2, 3, 4 Department of General Medicine, Government Medical College, Thiruvananthapuram, Kerala, India.
}

\section{ABSTRACT}

\section{BACKGROUND}

Organophosphorus poisoning is possibly the most common acute poisoning in the developing countries and is a global health problem.1,2 Between 750,000 and $3,000,000$ of OP poisoning occur globally every year. ${ }^{3}$ OP insecticides bind and irreversibly inhibit butyrylcholinesterase. These compounds cause excessive stimulation of muscarinic and nicotinic cholinergic receptors in the central and autonomic nervous systems and at skeletal neuromuscular junctions. ${ }^{4}$ Butyrylcholinesterase (BChE) is also known as pseudocholinesterase. ${ }^{5}$ Due to the limitations in accuracy and cost of plasma pseudocholinesterase level estimation, alternate biochemical marker options for OP poisoning have been studied for evaluation of patients with OP poisoning. Increased creatine phosphokinase (CPK) levels are seen in OP poisoning in early phase. ${ }^{6,7}$ This study was conducted to correlate initial serum CPK levels with the clinical severity at presentation and the final clinical outcome.

\section{METHODS}

This descriptive study was carried out after reviewing case records of patients $>/=$ 18 years with acute organophosphorus (OP) poisoning who were admitted under the Internal medicine department of Government Medical College Thiruvananthapuram between January 2019 and December 2019. The severity of poisoning was categorized based on Peradeniya organophosphorus poisoning (POP) scale. Serum CPK level, serum pseudocholinesterase, and total dose of atropine in milligrams until the outcome in the hospital were recorded. The student's t-test and Pearson's correlation coefficient were used for the assessment of statistical significance.

\section{RESULTS}

Clinical severity as per POP SCALE was mild (score 0-3) in 24 (27\%), moderate (score 4-7) in 50 (55.81\%) and severe (score 8-11) in 16 (18\%) patients. Serum $\mathrm{CPK}$, serum pseudocholinesterase level, and total atropine dose strongly correlated with clinical severity of OP poisoning. Our study strongly recommends serum CPK as an alternative marker.

\section{CONCLUSIONS}

Serum CPK level can be an effective biomarker in acute OP poisoning because its level can predict the prognosis of OP poisoning.

\section{KEY WORDS}

Creatine Phosphokinase, Organophosphorus Poisoning, Severity.
Corresponding Author: Dr. Regi David Sam, Associate Professor, Glory Bhavan Mariyapuram PO., Trivandrum-695122, Kerala, India. E-mail: regidavid1981@gmail.com

DOI: $10.14260 /$ jemds/2022/33

How to Cite This Article:

Chellappan AK, Sam RD, Pillai PR et al. Initial serum creatine phosphokinase level as an indicator of severity and prognosis of acute organophosphorus poisoning - a retrospective clinical study. J Evolution Med Dent Sci 2022;11(01):173-178, DOI: 10.14260/jemds/2022/33

Submission 06-01-2022,

Peer Review 13-01-2022,

Acceptance 23-01-2022,

Published 29-01-2022.

Copyright (c) 2022 Ajitha Kesi Chellappan et al. This is an open access article distributed under Creative Commons Attribution License [Attribution 4.0 International (CC BY 4.0)] 


\section{BACKGROUND}

Organophosphorus compounds are derived from phosphoric, phosphonic and phosphinic acids. ${ }^{8}$ They are widely used in agriculture and are easily available making them a frequent mode of self-harm. ${ }^{9}$ OP poisoning is possibly the most common acute poisoning in developing countries and is a global health problem.1,2 Between 750,000 and 3,000,000 OP poisoning occur globally every year. ${ }^{3}$

OP insecticides bind and irreversibly inhibit carboxylic ester hydrolases, such as acetylcholinesterase (AChE), erythrocyte cholinesterase (EChE), plasma or butyrylcholinesterase (BChE). These compounds cause excessive stimulation of muscarinic and nicotinic cholinergic receptors by the accumulated acetylcholine in the central and autonomic nervous systems and at skeletal neuromuscular junctions ${ }^{4}$. Butyrylcholinesterase (BChE) is otherwise referred to as pseudocholineesterase ${ }^{5}$. Reactive oxygen species level is also increased in OP poisoning resulting in oxidative stress causing DNA damage and cell death by cell membrane lipid peroxidation. ${ }^{10}$

Patients with OP poisoning are subjected to a cholinesterase test to evaluate the degree of systemic effects. Two enzyme levels are evaluated in OP poisoning. Plasma cholinesterase/Butyrylcholinesterase (BChE) levels indicate acute effects of organophosphate poisoning and is the widely used method.11,12,13,14 But the specificity is lesser than, the red blood cell cholinesterase level estimation. ${ }^{15,16}$ The red blood cell cholinesterase levels indicate long-term, or chronic, exposure, and are more specific. But estimation is more expensive and is not widely used. ${ }^{17}$

The significance of the alteration of these two enzymes is different. So evaluation of both enzyme levels gives an idea of the extent of the systemic effect of the poison. Significant differences have been noted in enzymatic testing methods as well as values between laboratories all across the globe. ${ }^{18}$ Estimation of EChE levels is costly and not regularly performed in most laboratories.19,20 Also a variety of pathologic conditions, physiological alterations and medications can lower plasma pseudocholinesterase activity.21,22,23,24

Due to the limitations in the accuracy of plasma pseudocholinesterase level estimation, alternate biochemical marker options for OP poisoning have been studied which can be used in conjunction with the enzyme level estimation for evaluation of patients with OP poisoning. Creatine phosphokinase (CPK) levels are being studied widely in patients presenting with OP poisoning. ${ }^{6}$ Increased CPK levels are seen in OP poisoning in early, intermediate, and chronic phases.6,7

\section{Objectives}

To correlate initial CPK levels with the clinical severity graded as per Paradeniya OP poisoning scale in patients presenting with acute organophosphorus poisoning.

\section{METHODS}

This retrospective clinical study was carried out reviewing the case files of patients $>/=18$ yrs. with acute organophosphorus (OP) poisoning who were admitted under internal medicine department of Government medical college Trivandrum. The case files of 92 patients admitted between January 2017 and December 2017 were reviewed.

The inclusion criteria were patients $>/=18$ yrs presenting within 6 hours of exposure to organophosphorus poison without previous treatment. The data collection for the study was started only after getting clearance from the ethical committee.

Diagnosis of OP poisoning was based on a) history of OP poisoning combined with characteristic symptoms \& signs of cholinergic toxidrome, toxicology evidence of gastric aspirate showing organophosphorus poison.

Exclusion criteria were: (i) patients below 18 years of age; (ii) patients who did not have the initial serum levels of AchE and creatine phosphokinase within 8 hours of presentation (iii) patients being exposed to other drugs or substances increasing cholinergic activity; (iv) chronic alcoholism; (v) presence of muscle /liver disease or chronic kidney disease (vi) epilepsy, (vii) known psychiatric illness, (viii) recent intramuscular injections, (ix) myocardial infarction, (x) myocarditis, (xi) trauma, (xii) malignancy or sepsis (xiii) new seizure and (xiv) drugs that may elevate creatine kinase (HMG-CoA reductase inhibitors (statins), fibrates, alcohol, D-penicillamine, interferon- $\alpha$, procainamide, chloroquine, hydroxychloroquine, quinacrine, perhexiline, amiodarone, emetine, ipecac, corticosteroids). ${ }^{25}$

A total of 150 cases conforming to the inclusion criteria were selected.

Demographic data (age, gender), alcohol consumption, history of psychiatric illness, medication history, features of cholinergic toxidrome, clinical severity according to the Peradeniya organophosphorus poisoning (POP) scale were noted. Blood investigations like blood sugar, blood urea, arterial blood gas analysis, serum creatinine, and ECG were recorded. The total atropine dose in "mg" required for the first three days, morbidity in terms of requirement of mechanical ventilation, final clinical outcome (complete recovery/ mortality) were compared with initial serum pseudocholinesterase level and serum CPK levels. BChE and CPK levels were estimated spectrophotometrically and lab reference values were - [4850 -12000 IU/I], ${ }^{26}$ CPK - [24 -195 $\mathrm{IU} / \mathrm{l}$ in males and $24-170 \mathrm{IU} / \mathrm{l}$ in females] ${ }^{27}$ respectively.

\section{Statistical Analysis}

The collected data was analysed using SPSS version 17 software (IBM SPSS Statistics). Categorical data were analysed and presented as percentages. Mean and standard deviation were used to express quantitative data.

The tests of significance used were Paired t-test, ANOVA (F) test and Spearman's correlation coefficient (rho). ROC curve was used to compare the accuracy of initial CPK levels and BChE levels in the diagnosis of acute OP poisoning. The accepted level of significance in this work was $0.05 ; \mathrm{P}<0.05$ was considered statistically significant. ${ }^{28}$ 
RESULTS

Case records of 92 patients were analysed in the study. Out of them, 62 were men $(67 \%)$ and 30 [32.6\%] were women (male: female) ratio was 2:1. They were aged from sixteen years to eighty-eight years (mean age $=35.5$ years). Fifty-one patients $(80.95 \%)$ came from a lower and lower-middle socioeconomic status. The majority of them were farmers by occupation; others were college students, businessmen, professionals and homemakers. Chlorpyriphos (28\%) was the most common poison consumed followed by triazophos and methyl parathion $16.6 \%$ each and the rest were combinations. The average time taken to reach the hospital was $286.26 \pm 92.53 \mathrm{~min}$.

Most common presentation was vomiting [60 (88.3\%)], followed by miosis [53 (81.95\%)], bradycardia [46 (70.84 $\%)]$, increased salivation [34 (60.84\%)], sweating [28 (49.97 $\%)]$, altered sensorium [27 (49.08\%)], tachypnea [15 (28.5 $\%)]$, muscle fasciculation [10 (10.03\%)], etc. Co-ingestion of alcohol was present in 25 [45.23\%] and history of psychiatric illness was noted in 9 [9.78\%] cases.

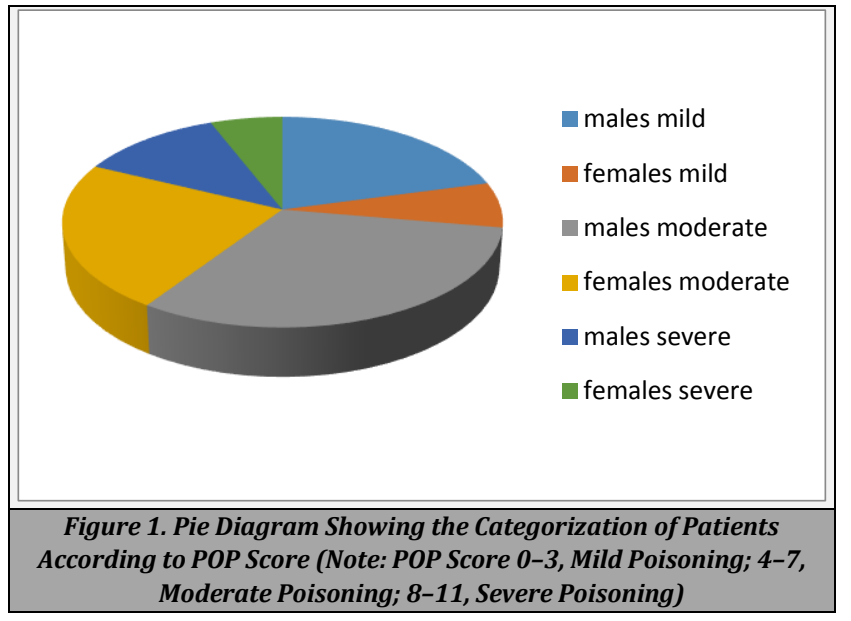

According to the POP score, 26 out of 92 patients (27\%), 50 out of $92(50.8 \%)$ and 16 out of 92 (18.2\%) were considered as mild, moderate and severe cases of poisoning, respectively [Figure 1]. Their serum pseudocholinesterase levels (in IU/L), the total dose of atropine (in $\mathrm{mg}$ ) in the first 3 days and serum CPK levels (in IU/L) are detailed in Table 1]. We observed that as the POP score increased, the serum creatine phosphokinase values also increased and there is an increase in the atropine requirement.

\begin{tabular}{|c|c|c|c|c|}
\hline $\begin{array}{l}\text { POP } \\
\text { Score }\end{array}$ & $\begin{array}{l}\text { No. of } \\
\text { Patients } \\
\text { (\%) }\end{array}$ & $\begin{array}{l}\text { PChE Levels } \\
\text { [IU/L]Mean } \\
\quad[ \pm \text { SD] }\end{array}$ & $\begin{array}{l}\text { Total Atropine } \\
\text { for } 3 \text { Days [mg] } \\
\text { Mean[+- SD] }\end{array}$ & $\begin{array}{c}\text { Initial CPK } \\
\text { Levels }[\mathrm{IU} / \mathrm{L}] \\
\text { Mean }[ \pm \mathrm{SD}]\end{array}$ \\
\hline $0-3$ & $26(27)$ & 7654.57 (1022.64) & $12.33(4.43)$ & 240.52 (102.61) \\
\hline $4-7$ & $50(55.2)$ & 4557 & & \\
\hline 8-11 & $16(18.8)$ & $1050.32(503.33)$ & $98.32(18.19)$ & $1123.65(210.08)$ \\
\hline \multicolumn{5}{|c|}{$\begin{array}{c}\text { Table 1. Peradeniya Organophosphorus Poisoning Scores, Mean } \\
\text { Serum Pseudocholinesterase Levels (IU/L), pH, a Total Dose of } \\
\text { Atropine (in mg) and Serum CPK Levels (IU/L) of the Patients on } \\
\text { Admission (N=63) }\end{array}$} \\
\hline
\end{tabular}

Initial CPK level in mild cases (POP SCORE $0-3$ ) was $240.5 \pm 102.61$ compared to $448.05 \pm 72.40$ in moderate poisoning and $1123.65 \pm 210.08 \mathrm{IU} / \mathrm{L}$ in severe poisoning (POP) 8 - 11. The total dose of atropine in mild cases of poisoning was $12.33 \pm 4.43 \mathrm{mg}$ while moderate severity required $50.06 \pm 10.06 \mathrm{mg}$ and severe cases required $98.32 \pm$ $18.19 \mathrm{mg}$. Pseudocholinesterase level showed declining trends in severe organophosphorus poisoning as evidenced by $7654.57 \pm 1022.64 \mathrm{I} \mathrm{U} / \mathrm{L}$ in mild cases and $4557.22 \pm$ 796.12 in moderate poisoning and $1050.32 \pm 503.33 \mathrm{I} \mathrm{U} / \mathrm{L}$ in severe cases. This indicated that there was a high degree of correlation between initial CPK value and POP score, serum pseudocholinesterase levels, and dose of atropine (in mg) [details shown in Table 2] The correlation was statistically significant $(\mathrm{P}<0.001)$ in each case.

\begin{tabular}{|cc|}
\hline Correlation between Variables & P-Value \\
Serum CPK and pseudocholinesterase & $<0.05$ \\
Serum CPK and POP scale & $<0.05$ \\
Serum CPK and dose of atropine in the first 3 days & $<0.05$ \\
\hline Table 2. Correlation between the Initial Creatine Phosphokinase \\
Value (in IU/L) and POP Score, Serum Pseudocholinesterase \\
\hline $\begin{array}{l}\text { POP= The Peradeniya organophosphorus poisoning scale, CPK= creatine } \\
\text { phosphokinase. }\end{array}$ \\
\hline
\end{tabular}

In our study, various complications like pulmonary oedema [10 (15.87 \%)], hypotension [7 (11.11\%)], altered sensorium, [7 (7\%)] seizures [5 (7.94\%)] and intermediate syndrome [5 (7.94\%)] were noted. We have observed that as the clinical severity of the poisoning increased with respect to Peradeniya organophosphorus poisoning (POP) score, the serum CPK levels and total atropine dose required for treatment also increased and the levels of serum pseudocholinesterase were reduced. Shapiro-Wilk normality test was applied for CPK distribution, it was not Gaussian distribution, probably the three patients with intermediate syndrome had a mean CPK (IU/L) of $1847.33 \pm 233.19$, and in those without intermediate syndrome was $214.81 \pm 139.22$. This difference in CPK levels in patients without IMS and with intermediate syndrome shows that there is a relationship between initial serum creatine phosphokinase and intermediate syndrome.

Initial CPK values [mean 1847.33 $\pm 233.19 \mathrm{IU} / \mathrm{L}$ ] were high in patients with intermediate syndrome and these three patients expired. Ten out of 92 patients $(10.86 \%)$ expired in our study with males 6 [60\%] and females 4 [40\%]. It was found that mortality was high in patients with high initial CPK values. The cause of death included respiratory paralysis, metabolic acidosis, acute respiratory distress syndrome (ARDS), acute renal failure, CNS depression, or a combination of these. Two patients with intermediate syndrome expired.

\section{DISCUSSION}

Organophosphorus poisoning is one of the leading causes of high morbidity and mortality in developing countries. Timely recognition and prompt management are very critical in the outcome. Since OP compounds are widely used for agricultural and industrial purposes, they are becoming a major source of health hazards. So, it is crucial to recognize the entire spectrum of the symptoms. Symptoms are mainly classified into muscarinic and nicotinic based on the receptor involved. Muscarinic features include excessive salivation, lacrimation, urination, diarrhoea, abdominal cramps, emesis, pinpoint pupils, bradycardia, and wheeze. Nicotinic features include fasciculation, paralysis, hypertension and tachycardia. Central receptor features include anxiety, 
confusion, seizures, psychosis and ataxia. Three types of paralysis are observed.29,30,14 Type I is caused by continued depolarization at neuromuscular junction whereas type II is secondary to intermediate syndrome and type III is due to delayed polyneuropathy. Our study has demonstrated that initial serum creatine phosphokinase is increased in severe organophosphorus poisoning which is in concordance with Bhattacharyya et al. who described a high degree of correlation between initial CPK value and POP scale, serum EChE levels and total dose of atropine in acute OP poisoning. Their study showed that in a significant number of patients with clinically severe poisoning, CPK levels were elevated even in the absence of intermediate syndrome, presumably due to muscle fibre necrosis, confirmed by muscle biopsy in 2 of their patients. Our study is in concordance with the same finding that serum CPK level is elevated even in the absence of intermediate syndrome the half-life of CPK is about 1.5 days. Therefore in moderate to severe OP poisoning, if the muscle fibres undergo a single insult, it normalizes within 56 days 34. CPK evaluation is cheap and easily quantifiable. Serum CPK can be used in predicting as well as assessing the prognosis of patients with OP poisoning. Muscle fibre necrosis in OP poisoning has been demonstrated in animal experimental studies by Calore et al. ${ }^{25}$ Sharma et al. has observed that serum creatine phosphokinase was elevated in a fraction of their cases who had severe poisoning, rhabdomyolysis was observed in "intermediate syndrome" and lead to raised CPK level. ${ }^{26}$ The three types of muscle injuries occurring in OP poisoning are (i) Type I is due to continuous depolarization at neuromuscular junction presumably causing muscle fibre injury (occurring during the initial cholinergic crisis), (ii) type II due to intermediate syndrome (due to rhabdomyolysis) and (iii) type III due to delayed polyneuropathy. ${ }^{31,32}$ Intermediate syndrome occurs in patients 24-96 hours after acute OP poisoning.,432,33 Perreault et al. 38 observed that skeletal muscle injury promptly caused CPK to leak into the blood and urine making it the best biomarker for detecting and monitoring skeletal muscle damage. ${ }^{34}$ In the case of a single insult to the muscle, CPK normalizes within 5-6 days. CPK levels peak within 24 to 48 hours of the onset of muscle injury or rhabdomyolysis and then decline at the relatively constant rate of $39 \%$ of the previous day's value. ${ }^{35,36}$ As in the study done by Geller et al., our study also demonstrated that the more severe the poisoning, the total amount of atropine required for treatment increased dramatically. It has been found that perinatal asphyxia with acidosis causes leakage of CPK from the damaged cells into the circulation and that a marked elevation of CPK levels may indicate a poor prognosis. ${ }^{37}$ Therefore CPK can be falsely high in patients with acidosis in OP poisoning. Respiratory and metabolic acidosis is relatively a common complication in acute OP poisoning. So serum creatine phosphokinase levels should be used with caution in patients with metabolic and respiratory acidosis in organophosphorus poisoning. Sahjian and Frakes ${ }^{23}$ stated that if there is an ongoing injury to the muscle due to the development of complications, the CPK level continues to be elevated. The increased muscle injury warrants an increased need for early ventilator care. Thus, keeping the half-life of CPK in view, a repeat measurement of CPK level after 48 hours will help in early identification of ongoing muscle injury, need for early ventilator care and improve the prognosis. In the present study, the correlations between initial serum CPK levels at admission showed a high degree of positive correlation with ventilator support and outcome of the patient $(\mathrm{r}=0.686,0.578$ and 0.525$)$. These correlations were also statistically significant $(\mathrm{P}=0.001)$. Hence, we recommend the usefulness of estimating CPK levels, especially after 48 hours, so that complications can be recognized at the earliest and patients can be immediately managed, reducing morbidity and mortality in patients with organophosphorus poisoning averting the use of mechanical ventilation. ${ }^{12}$ Majority of patients in our study were in the age group of 21 - 40 years [mean age 35.5 years]. This is in consensus with the Bhattacharyya's1 study which showed most patients in the age group of $21-40$ years. A study done by Raghavendra Mural et al. and by Kinathankaraiyan Nagarajan et al. showed similar results. The majority of the patients in our study were in the third decade and would contribute economy for the livelihood of their family. Senanayeke et al. has observed that the POP score can efficiently predict the severity, morbidity and mortality of OP poisoned patients. ${ }^{38}$ This scale uses a high respiratory rate $(>$ 20 / minute) and the presence of cyanosis. This approach is likely to cause difficulties as severe OP poisoning may cause central respiratory depression with a reduced respiratory rate or tachypnea in the context of increased respiratory secretions, bronchoconstriction or respiratory muscle weakness ${ }^{39,40,41}$ but, in our study, we found that two patients with a POP score less than 3 had expired showing that POP scores can be deceptive and even a person with relatively low POP score can develop complications or expire prematurely.

The pursuit for newer biomarkers in relation to OP poisoning started quite a long time back. Some researchers have proposed OP-labelled albumin in human plasma 28,33 and blood $\beta$-glucuronidase. Measurement of paraoxonase $36,42,43$ status has been suggested as a reliable marker to assess susceptibility to OP poisoning. In children, meconium and urine have been studied for estimation of biomarkers indicating susceptibility to chronic OP exposure in utero. ${ }^{44}$ Since a small number of human subjects were involved in these studies, they might not be so reliable when applied to large populations. The estimated cost of these biomarkers is high limiting their use in developing countries.

\section{CONCLUSIONS}

So, to conclude due to the relatively low cost, easy availability and high degree of correlation with mortality, initial serum CPK can be proposed as an emerging marker for predicting the severity and prioritising the patients for aggressive management. However, the main limiting factor with CPK is its non-specificity. So, other causes of raised CPK should be excluded before interpreting serum creatine phosphokinase in patients of acute OP poisoning. Further studies with more patients and histopathological studies including muscle biopsy are required to support our observation. Our study was carried out with a relatively small number of patients, and in only one centre located in Southern India, so we might be prejudiced about the association, efficacy and reliability of CPK levels on the severity of OP poisoning among other ethnic groups. 
Data sharing statement provided by the authors is available with the full text of this article at jemds.com.

Financial or other competing interests: None.

Disclosure forms provided by the authors are available with the full text of this article at jemds.com.

\section{REFERENCES}

[1] Buyukokuroglu ME, Cemek M, Tosun M et al, Dantrolene may prevent organophosphate-induced oxidative stress and muscle injury. Pesti Biochem Physiol 2008;92(3):156-63.

[2] Liu JH, Chou C, Liu YL et al. Acid-base interpretation can be the predictor of outcome among patients with acute organophosphate poisoning before hospitalization. Am J Emerg Med 2008;26(1):24-30.

[3] Palaniappen V, Muruganathan A, Geetha T, et al. Current concepts in the management of organophosphorus compound poisoning. Chap- 95, API Update 2013.

[4] Clark RF, Insecticides: Organic phosphorus compounds and carbamates. Chap- 109. In: Flomenbaum NE, Goldfrank LR, Hoffman RS, et al, eds. Goldfrank's toxicologic emergencies. $8^{\text {th }}$ edn. New York: McGraw-Hill 2006:1497-513.

[5] Maiorana A, Roach RB. Heterozygous pseudocholinesterase deficiency: a case report and review of the literature. J Oral Maxillofacial Surg 2003;61(7):845-7.

[6] Bhattacharyya K, Phaujdar S, Sarkar R, et al. Serum creatine phosphokinase: a probable marker of severity in organophosphorus poisoning. Toxicol Int 2011;18(2):117-23.

[7] Agarwal SB, Bhatnagar VK, Agarwal A, et al. Impairment in clinical indices in acute organophosphate insecticide poisoning patients in India. Internet J Toxicol 2007;4:1-6.

[8] Terry AV. Functional consequences of repeated organophosphate exposure: potential non-cholinergic mechanisms. Pharmacology Therapeutics 2012;134(3):355-65.

[9] Sungur M, Güven M. Intensive care management of organophosphate insecticide poisoning. Crit Care 2001;5(4):211-5.

[10] Uzunhisarcikli M, Kalender Y. Protective effects of vitamins $\mathrm{C}$ and $\mathrm{E}$ against hepatotoxicity induced by methyl parathion in rats. Ecotoxicol Environmen Safety 2011;74(7):2112-8.

[11] Eddleston M, Eyer P, Worek F, et al. Predicting outcome using butyrylcholinesterase activity in organophosphorus pesticide self-poisoning. QJM 2008;101(6):467-74.

[12] Yamashita M, Tanaka J, Ando Y, et al. Human mortality in organophosphate poisonings. Vet Hum Toxicol 1997;39(2):84-5

[13] Worek F, Diepold C, Eyer P. Dimethylphosphorylinhibited human cholinesterases: inhibition, reactivation, and aging kinetics. Arch Toxicol 1999;73(1):7-14.

[14] Khan S, Hemalatha R, Jeyaseelan L, et al. Neuroparalysis and oxime efficacy in organophosphate poisoning: a study of butyrylcholinesterase. Hum Exp Toxicol 2001;20(4):169-74.
[15] Aygun D, Doganay Z, Altintop L, et al. Serum acetylcholinesterase and prognosis of acute organophosphate poisoning. J Toxicol Clin Toxicol 2002;40(7):903-10.

[16] Brahmi N, Mokline A, Kouraichi N, et al. Prognostic value of human erythrocyte acetylcholinesterase in acute organophosphate poisoning. Am J Emerg Med 2006;24(7):822-7.

[17] Jane P. Commercial pesticide applicators may get mandatory blood tests. Agrichemical Age March 1987

[18] Cholinesterase Inhibition. Extension Toxicology network, a pesticide information project of cooperative extension offices of Cornell University, Michigan State University, Oregon State University, and University of California at Davis. Publication Date: 9/93,

[19] Bazire A, Gillon E, Lockridge O, et al. The kinetic study of the inhibition of human cholinesterases by demeton- Smethyl shows that cholinesterase-based titration methods are not suitable for this organophosphate. Toxicol In Vitro 2011;25(3):754-9.

[20] Hassan NAM, Madboly AG. Correlation between serum creatine phosphokinase and severity of acute organophosphorus poisoning: a prospective clinical study (2012-2013). Journal of Environmental Science, Toxicology and Food Technology 2013;4(5):18-29.

[21] Leadingham CL. A case of pseudocholinesterase deficiency in the PACU. J Perianesth Nurs 2007;22(4):265-71.

[22] Soliday FK, Conley YP, Henker R. Pseudocholinesterase deficiency: a comprehensive review of genetic, acquired, and drug influences. AANA J 2010;78(4):313-20.

[23] Brozović G, Sunko BM, Hafner T, et al. Allergic reaction to suxamethonium during emergency caesarean section and pseudocholinesterase deficiency in the same patient. Wien Klin Wochenschr 2014;126(13-14):435-8.

[24] Lurati AR. Organophosphate exposure with pseudocholinesterase deficiency. Workplace Health Saf 2013;61(6):243-5.

[25] Calore EE, Sesso A, Puga FR, et al. Sarcoplasmic lipase and non-specific esterase inhibition in myofibers of rats intoxicated with the organophosphate isofenphos. Exp Toxicol Pathol 1999;51(1):27-33.

[26] De Wilde V, Vogelaers D, Colardyn F, et al. Postsynaptic neuromuscular dysfunction in organophosphate induced intermediate syndrome. Klin Wochenschr 1991;69(4):177-83.

[27] Panteghini M, Bais R, Solinge WW. Enzymes. Chap- 21. In: Burtis CA, Ashwood ER, Bruns DE, eds. Tietz Textbook of clinical chemistry and molecular diagnostics. $4^{\text {th }}$ edn. Philadelphia: Elsevier Saunders 2006:597-643.

[28] Peeples ES, Schopfer LM, Duysen EG, et al. Albumin, a new biomarker of organophosphorus toxicant exposure, identified by mass spectrometry. Toxicol Sci 2005;83(2):303-12.

[29] Lee P, Tai DY. Clinical features of patients with acute organophosphate poisoning requiring intensive care. Intensive Care Med 2001;27(4):694-9.

[30] Bardin PG, van Eeden SF, Moolman JA, et al. Organophosphate and carbamate poisoning. Arch Intern Med 1994;154(13):1433-41. 
[31] Aaron CK. Organophosphates and carbamates. Chap102. In: Ford MD, Delaney KA, Ling LJ, et al, eds. Clinical toxicology. $1^{\text {st }}$ edn Philadelphia: W.B. Saunders Co., 2001:983-991.

[32] Yang CC, Deng JF. Intermediate syndrome following organophosphate insecticide poisoning. J Chin Med Assoc 2007;70(11):467-72.

[33] Li B, Ricordel I, Schopfer LM, et al. Detection of adduct on tyrosine 411 of albumin in humans poisoned by dichlorvos. Toxicol Sci 2010;116(1):23-31.

[34] Schneider CM, Dennehy CA, Rodearmel SJ, et al. Effects of physical activity on creatine phosphokinase and the isoenzyme creatine kinase-MB. Ann Emerg Med 1995;25(4):520-4.

[35] Sahjian M, Frakes M. Crush injuries: pathophysiology and current treatment. Nurse Pract 2007;32(9):13-8.

[36] Costa LG, Cole TB, Vitalone A, et al. Measurement of paraoxonase (PON1) status as a potential biomarker of susceptibility to organophosphate toxicity. Clin Chim Act 2005;352(1-2):37-47.

[37] Warburton D, Singer DB, Oh W. Effects of acidosis on the activity of creatine phosphokinase and its isoenzymes in the serum of newborn infants. Pediatrics 1981;68(2):195-7.
[38] Senanayake N, De Silva HJ, Karalliedde LA. Scale to assess severity in organophosphorus intoxication: POP scale. Hum Exp Toxicol 1993;12(4):297-9.

[39] Eddleston M, Mohamed F, Davies JOJ, et al. Respiratory failure in acute organophosphorus pesticide selfpoisoning. QJM 2006;99(8):513-22.

[40] Pore NE, Pujari KN, Jadkar SP. Organophosphorus Poisoning. IJBPs 2011;2:606-12.

[41] Sniderman AD. Is there value in liver function test and creatine phosphokinase monitoring with statin use? Am J Cardiol 2004;94(9A):30F-4.

[42] Soltaninejad K, Shadnia S, Afkhami-Taghipour M, et al. Blood $\beta$-glucuronidase as a suitable biomarker at acute exposure of severe organophosphorus poisoning in human. Hum Exp Toxicol 2007;26(12):963-6.

[43] Sirivarasai J, Kaojarern S, Yoovathaworn K, et al. Paraoxonase (PON1) polymorphism and activity as the determinants of sensitivity to organophosphates in human subjects. Chem Biol Interact 2007;168(3):184-92.

[44] Wessels D, Barr DB, Mendola P. Use of Biomarkers to Indicate Exposure of children to organophosphate pesticides: Implications for a longitudinal study of children's environmental health. Environ Health Perspect 2003;111(16):1939-46. 